\title{
Sesgos de las historias de la literatura: tres géneros narrativos populares en español y sus fuentes
}

\author{
Bias in Literary History: Three Popular Narrative Genres in \\ Spanish and their Sources
}

\author{
Daniel Gutiérrez Trápaga \\ Universidad Nacional Autónoma de México \\ danielgutierrez@filos.unam.mx
}

\section{Resumen}

Este trabajo trata sobre la presencia y presentación de tres géneros populares en las historias de la literatura latinoamericana y española que se remontan, por lo menos, al siglo XIX y continúan en la actualidad: el gótico, la fantasía y la ciencia ficción. En particular, se revisa la tendencia a omitir o minimizar dichos géneros; en contraste con la información obtenida de las fuentes primarias, la cual demuestra una importante presencia de estos tres géneros en la literatura escrita en español.

Palabras clave: historia de la literatura, metodología, gótico, fantasía, ciencia ficción.

\begin{abstract}
This work examines the presence and presentation of gothic, fantasy and science fiction in several histories of Spanish and Latin-American literature. These three genres date back to at least the nineteenth century and they remain ever so popular. In particular, this work focuses on a trend that systematically either omits or minimizes these genres. This trend is in sharp contrast with the picture obtained from primary sources and it allows to demonstrate a strong presence of the aforementioned genres in in Spanish.
\end{abstract}

Keywords: history of literature, methodology, gothic, fantasy, science fiction.

Recibido: $15 / 04 / 2020$

Aceptado: 02/03/2021 


\section{Distant Reading (lectura distante) y sesgos autorales: la metodología de la historia de la literatura}

Uno de los problemas fundamentales de la investigación literaria surge de la finitud de la vida humana, lo que imposibilita que una sola persona sea capaz de leer la enorme producción literaria, no sólo a nivel mundial, sino lingüístico, nacional o en un período dado. Dicha condición de índole cuantitativa llevó a Franco Moretti a proponer una nueva técnica de lectura e investigación literaria: distant reading o lectura distante. Tal propuesta se encuentra en el extremo opuesto de la técnica de lectura que sirve de punto partida a buena parte de la investigación y la docencia literaria: close reading o lectura atenta. Luego, el empleo del distant reading implica un manejo de las fuentes literarias que privilegia lo cuantitativo, desplazando la individual del texto que prima con el close reading:

But the trouble with close reading ... is that it necessarily depends on an extremely small canon. This may have become an unconscious and invisible premise by now, but it is an iron one nonetheless: you invest so much in individual texts only if you think that very few of them really matter. Otherwise, it doesn't make sense. And if you want to look beyond the canon. . ., close reading will not do it. lt's not designed to do it, it's designed to do the opposite. At bottom, it's a theological exercise very solemn treatment of very few texts taken very seriously whereas what we really need is a little pact with the devil: we know how to read texts, now let's learn how not to read them. Distant reading: where distance, let me repeat it, is a condition of knowledge: it allows you to focus on units that are much smaller or much larger than the text: devices, themes, tropes-or genres and systems. And if, between the very small and the very large, the text itself disappears, well, it is one of those cases when one can justifiably say, less is more. If we want to understand the system in its entirety, we must accept losing something (Moretti, "Conjectures on World Literature" 48-49).

El trabajo de Moretti se enfoca primordialmente en el estudio de la literatura universal, donde la magnitud del problema del manejo de fuentes es central y obvia; sin embargo, las dificultades de esta índole no son ajenas a la investigación de las literaturas nacionales. Por ejemplo, el propio Moretti ha reflexionado sobre los problemas cuantitativos en los estudios sobre la novela inglesa decimonónica: 
The majority of books disappear forever -and 'majority' actually misses the point: if we set today's canon of nineteenth-century British novels at two hundred titles (which is a very high figure), they would still be only about 0.5 per cent of all published novels. And the other 99.5 per cent? This is the question behind this article, and behind the larger idea of literary history that is now taking shape in the work of several critics ("The Slaughterhouse of Literature" 66).

Esta última perspectiva, la de la revisión amplia de fuentes, sus problemas y técnicas de investigación, en contraste con los sesgos de la perspectiva puramente textual, es la que interesa a este artículo, pero enfocada en el caso particular de la narrativa en español.

Este trabajo examina la información panorámica en diversas historias de la literatura reciente de algunos géneros populares en español para contrastarla con la información cuantitativa de fuentes primarias. Las historias de la literatura resultan idóneas para examinarlas a partir del distant reading por su naturaleza amplia y el afán de abarcar un corpus vastísimo, el de una literatura nacional o regional y, en el caso de este trabajo, lingüística y transnacional. Los trabajos de historia de la literatura inevitablemente responden a una serie de decisiones y factores de diversa índole: ideológicos, lingüísticos, nacionales, raciales, estéticos, políticas, pragmáticos, materiales, etc. A partir de estos dispares factores, muchas veces implícitos, se conforman los criterios de evaluación y selección de las fuentes literarias dentro de la inmensidad de la producción literaria de una nación, región o lengua para conformar las historias de la literatura (Perkins, Sumillera). Sin importar su amplitud, estos trabajos están imposibilitados para abarcar por completo su objeto de estudio y tienen a la síntesis de las fuentes como un principio estructural. Luego, interesa particularmente los factores metodológicos y los sesgos que estos conllevan en las tareas titánicas que conllevan este tipo de estudios.

A pesar de la inmensidad de su tarea, las historias de la literatura se han construido, paradójicamente, a partir de técnicas de investigación basadas en el close reading. Prueba de esto es que domina la perspectiva del autor sobre la de los géneros en las historias de la literatura, como señaló Roland Barthes:

Je dis seulement que le temps de la synthèse est venu, mais que cette synthèse ne pourra jamais s'accomplir dans les cadres actuels de l'histoire littéraire. Derrière ces lacunes, en effet, il y a un vice qui, pour n'être que de point de vue, et non d'information, n'en est pas moins fondamental: le privilège “centralisateur" accordé à l'auteur (528). 
Es decir, existe un sesgo metodológico al aplicar una técnica para abarcar un corpus breve, cuando las historias de la literatura tienen que abarcar corpus gigantescos, que sobrepasan la capacidad lectora de una vida humana. Luego, la revisión de este género académico desde el distant reading permite trascender el límite de la figura autoral y observar con mayor amplitud el objeto de estudio, incluyendo los asuntos cuantitativos en los que se enmarca la configuración de los géneros literarios. Luego, existe la posibilidad de replantear los criterios de selección de fuentes. Con esta propuesta, se busca mostrar importantes sesgos y puntos ciegos, derivados de técnicas y perspectivas puramente cualitativas y subjetivas, en los estudios panorámicos actuales de la novela y el cuento, tanto latinoamericano como español, y en la configuración de su canon.

Para realizar dicho estudio se seleccionó una muestra representativa de las historias de la literatura reciente, publicadas del año 2000 en adelante. El criterio cronológico busca mostrar las tendencias más recientes en las que los estudios panorámicos y diacrónicos describen los géneros, modos y el campo de la literatura escrita en español. En particular, este trabajo se enfoca en tres géneros narrativos populares, de gran presencia en la cultura contemporánea: el gótico (o el horror para apelar a una definición amplia), la ciencia ficción y la fantasía. Es precisamente en las últimas décadas que han surgido importantes trabajos especializados en torno a dichos géneros y, sin embargo, las aportaciones de tales estudios permanecen, en su mayoría, ignorados en las obras de carácter general. 
Los géneros populares en la literatura occidental surgidos desde finales del siglo XVIII, el gótico, ${ }^{1}$ la fantasía ${ }^{2}$ y la ciencia ficción, ${ }^{3}$ entendidos de manera amplia, comparten un rasgo poético central en su configuración: no pertenecen al paradigma mimético o realista de la ficción. Por ello, son géneros afines cuya poética e historia está profundamente interrelacionada (Roberts 33-34). Además, son géneros con un alto potencial híbrido por lo que no es extraño encontrar textos que conjuntan elementos (temas, tópicos y motivos) de dos o tres de estos géneros, en la medida en que los tres se alejan del paradigma de realidad del lector y suelen contener elementos sobrenaturales. En ese sentido, el presente artículo defiende que la omisión del gótico, la ciencia ficción y la fantasía en los trabajos panorámicos depende más de su poética que de sus fuentes primarias y presencia real en la historia de la ficción en español. Luego, la información panorámica y cuantitativa sobre la existencia continua de fuentes de estos géneros, obtenida a partir de una

1 “... a Gothic tale usually takes place ... in an antiquated or seemingly antiquated space... Within this space, ... are hidden some secrets from the past (sometimes the recent past) that haunt the characters ... These hauntings can take many forms, but they frequently assume the features of ghosts, specters, or monsters (mixing features from different realms of being, often life and death) that rise from within the antiquated space ... Gothic fictions generally play with and oscillate between the earthly laws of conventional reality and the possibilities of the supernatural ... often siding with one of these over the other in the end, but usually raising the possibility that the boundaries between these may have been crossed" (Hogle 2-3).

2 No me refiero a lo fantástico como Kafka y Borges, sino a la fantasía como las novelas de Tolkien, de quien tomo la definición: "I am thus not only aware but glad of the etymological and semantic connections of fantasy with fantastic: with images of things that are not only 'not actually present', but which are indeed not to be found in our primary world at all, or are generally believed not be found there. But while admitting that, I do not assent to the depreciative tone. That the images are of things not in the primary world (if that is indeed possible) is a virtue not a vice. Fantasy (in this sense) is, I think, not a lower but a higher form of Art, indeed the most nearly pure form, and so (when achieved) the most potent. Fantasy, of course, starts out with the advantage: arresting strangeness" (60). Para una aproximación teórica véase (Mendlesohn).

3 "Science fiction has proved notoriously difficult to define. It has variously been explained as a combination of romance, science, and prophecy (Hugo Gernsback), 'realistic speculation about future events' (Robert Heinlein), and a genre based on an imagined alternative to the reader's environment (Darko Suvin). Firstly, to call science fiction (SF) a genre causes problems because it does not recognize the hybrid nature of many SF works. It is more helpful to think of it as a mode or field where different genres and subgenres intersect. And then there is the issue of science ... Technology has repeatedly been associated with the future by SF, but it does not follow that the fiction is therefore about the future ... it is a 'What If Literature' ... It is helpful to think of an SF narrative as an embodied thought experiment whereby aspects of our familiar reality are transformed or suspended" (Seed 1-2). Para los propósitos de este trabajo la distinción teórica entre género y modo es poco relevante, pues lo que interesa destacar es la existencia de una enorme y longeva tradición de textos en español de la ciencia ficción, el gótico y la fantasía. Véase, para la ciencia ficción, también el planteamiento de Moreno desde el género literario. 
lectura distante, servirá como argumento para señalar la necesidad de su inclusión, mostrando las tendencias y sesgos de las metodologías y técnicas de investigación empleadas para construir la mayor parte de las historias de las literaturas. A partir de esto, se evidenciarán problemas en torno al manejo y descripción de fuentes primarias que deben ser zanjados para poder ampliar los estudios desde la perspectiva del distant reading y los géneros literarios y no puramente desde el canon y lo autoral.

\section{El gótico, la fantasía y la ciencia ficción en la historia de la literatura}

Un rasgo común de casi todas las obras y manuales dedicados a la ficción en español es el no reconocer la existencia de tres de los principales géneros no miméticos y realistas surgidos a partir del siglo XVIII, el gótico, la ciencia ficción y la fantasía. ${ }^{4} \mathrm{Al}$ no identificar los géneros en cuestión, la mayor parte de las obras que los conforman son sistemáticamente omitidas, lo que se refleja en primera instancia en el aspecto léxico. Comienzo con el caso de la literatura gótica para analizar la situación de estos tres géneros. Hace ya varias décadas, Julio Cortázar identificó la existencia de una clara tradición gótica en la literatura argentina y uruguaya:

Para desconcierto de la crítica, que no encuentra una explicación satisfactoria, la literatura rioplatense cuenta con una serie de escritores cuya obra se basa en mayor o menor medida en lo fantástico, entendido en una acepción muy amplia que va de lo sobrenatural a lo misterioso, de lo terrorífico a lo insólito, y donde la presencia de lo específicamente "gótico" es con frecuencia perceptible. Algunos célebres relatos de Leopoldo Lugones, las atroces pesadillas de Horacio Quiroga, lo fantástico mental de Jorge Luis Borges, los artificios a veces irónicos de Adolfo Bioy Casares, la extrañeza en lo cotidiano de Silvina Ocampo y del que esto escribe, y, last but not least, el universo surreal de Felisberto Hernández, son algunos ejemplos suficientemente conocidos por los amantes de esta literatura, quizá la única, dicho sea de paso, que admite ser calificada de escapista stricto sensu y sin intención peyorativa (Cortázar 145).

4 Una gran excepción es el trabajo de Oviedo, que reconoce la existencia de una tradición literaria distinta a la del realismo y los géneros no mimético; aunque los presente a todos como géneros subordinados a la literatura fantástica: "La literatura fantástica ocupa un espectro bastante amplio de formas cuyos límites extremos serían, por un lado, los relatos de horror y, por otro, la llamada "ciencia ficción"; es decir, las figuraciones aterradoras de lo ancestral o primario, y la anticipación de un futuro tecnológico amenazante o deshumanizador. En el área del Río de la Plata el cultivo de esta tendencia fue más abundante que en ninguna otra parte, aunque haya importantes expresiones en áreas distintas, como México y otros países" (Historia de la literatura hispanoamericana 4 39). 
Como se aprecia en la cita, la lista de autores elaborada por Cortázar no es desconocida, al contrario, es canónica. El empleo del término gótico aparece claramente entendido como una literatura distinta al realismo ("escapismo"), aunque con la enfática y reveladora aclaración de que esto no debe interpretarse de manera peyorativa. Además, el fragmento muestra el desconcierto crítico para tratar con dicha tradición.

A pesar de que el célebre autor argentino mostró una clara tradición literaria de corte gótico hace varias décadas, múltiples historias de la literatura recientes aún no lo hacen, si bien muchas reconocen y dedican secciones a los autores mencionados por Cortázar. Varias obras recientes e influyentes, publicadas en el ámbito angloparlante, como el A Companion to Latin American Literature de Stephen M. Hart, A Companion to Latin American Literature and Culture editado por Sara Castro-Klaren, la Modern Latin American Literature. A Very Short Introduction de González Echevarría dedican espacio a discutir varios o todos los autores mencionados por el argentino, pero sin describirlos como "góticos". De hecho, estos trabajos ni siquiera emplean esta palabra o términos cercanos como "horror" o "terror".

El caso de Horacio Quiroga resulta paradigmático para ilustrar la manera en que se evita utilizar las etiquetas genéricas precisas, aunque las descripciones sean claras. Por ejemplo, González Echevarría presenta así los cuentos de Quiroga: "The ambience of perversion, the ghoulishness, the presence of disease of both mind and body permeate Quiroga's fiction and reveal the pervasive influence of Edgar Allan Poe" (84). Las descripciones de los cuentos del uruguayo remiten a elementos constitutivos del gótico, pero la filiación genérica no es explícita. Además, y al igual que Cortázar, se reconoce la deuda con Poe, uno de los autores más paradigmáticos de dicho género, igual que lo hace Hart:

What is perhaps most interesting about Quiroga's short stories is their combination of the close attention to the here and now, described often with a scientific objectivity, with a hint of the supernatural, a device which Quiroga may well have learned from his master, Edgar Allan Poe, but which he transforms to produce a narrative unmatched in its use of suspense (175).

Nuevamente, se reconoce el vínculo con uno de los grandes maestros del género, pero sin asociarlo directamente con el gótico, al evitar el término. La gran excepción es la Historia de Oviedo, quien reconoce la deuda con Poe, pero no duda en asociar abiertamente la obra de Quiroga con el género del horror: 
... el dominante influjo que Poe ejerció sobre él desde sus inicios ... Ambos ['El almohadón de plumas' y 'La gallina degollada'] son 'cuentos de horror', elaborados con un solo efecto - o efectismo- en mente: sorprender al lector estremeciéndolo con un final chirriante... (Historia de la literatura hispanoamericana 3 21).

La tendencia dominante a evitar las etiquetas genéricas, "gótico" u "horror", no es privativa de los estudios de la literatura rioplatense o de la investigación en inglés, pues el género también tuvo desarrollo en otras geografías. Dicho sesgo aparece igualmente en los estudios panorámicos dedicados a la literatura española, ignorando el conocimiento aportado por los estudios especializados los cuales han demostrado una gran presencia del gótico en España. Trabajos de la última década como los de Miriam López Santos y los de Xavier Aldana Reyes muestran la llegada de decenas de relatos góticos a España, casi todos traducidos y adaptados del francés, desde las últimas décadas del siglo XVIII y durante el XIX. Además, desde inicios del XIX y hasta la fecha, se han escrito numerosas novelas y cuentos originales, en cuya lista de autores se encuentran figuras centrales como Cadalso, Espronceda, Bécquer, Alarcón, Clarín Pardo Bazán o grandes best-sellers del presente siglo, como las novelas de Ruiz Zafón y su tetralogía El cementerio de los libros olvidados (2001-2016). Si bien este recuento no es exhaustivo, estamos ante centenas de fuentes y más de dos siglos de existencia, tan sólo en el siglo XIX contamos con 54 novelas góticas que circularon en España y decenas de cuentos (López Santos). ${ }^{5}$ A pesar de esto, la existencia del género en español no aparece consignada en las historias de la literatura recientes, como las de Turner y López de Martínez; Alonso; Gracia y Ródenas; González Echevarría o Mainer. ${ }^{6}$

Para describir obras individuales, las menciones al gótico son muy escasas. En ese sentido, Labanyi utiliza el término "Gothic" para describir el desenlace de El estudiante de Salamanca de Espronceda y las Noche lugubres de Cadalso (Labanyi 4 y 58). Por su parte, la introducción al A Companion to the Twentieth-century Spanish Novel reconoce la influencia del género en su introducción, aunque en el resto del texto no se vuelva a mencionar: "Spanish fiction has breathed new life into some

5 Para una revisión detallada de las fuentes primarias del gótico en España, véase (Gutiérrez Trápaga, "La narrativa gótica en la historia de la literatura española...").

6 Dos notables excepciones son los capítulos sobre la prosa del XVIII de Álvarez Barrientos en la Cambridge History of Spanish Literature y la History of the Spanish Novel, donde identifica los orígenes y la importancia del gótico en la época. Desafortunadamente, los estudios de los períodos subsecuentes en dichas obras no dan continuidad a la presencia del gótico en la literatura española, al omitir por completo su existencia más allá del siglo XVIII. 
less frequented and non-native traditions: science fiction, the Byzantine novel, the Gothic novel, and magical realism" (Altisent, "Introduction" 13). De igual manera, Hart emplea el adjetivo "gótico" para describir "La muñeca menor" de Rosario Ferré y para describir una de las tres tradiciones narrativas presentes en Luna caliente de Mempo Giardinelli (235 y 254). El vocablo "gótico" apenas se emplea en el capítulo dedicado a las insurrecciones femeninas del siglo XX para señalar uno de los múltiples elementos que confluyen en Cartucho (1931) de Nelli Campobello en el Companion to Latin American Literature and Culture (Bergero y Marchant 522). Por su parte, Espinasa emplea el término para adjetivar algunos relatos de Francisco Tairo, Carlos Fuentes, Guadalupe Dueñas y la novela Karpus Minthej de Jordi García Bergua (219, 224, 315 y 351). Finalmente, hay que destacar los recientes volúmenes colectivo editados por Justin D. Edwards y Sandra Guardini Vasconcelos, y por Sandra Casanova-Vizcaíno e Inés Ordiz, que, si bien no se tratan de historias de la literatura, ni pretenden serlo, abren nuevas líneas de estudios y establecen firmemente la existencia del género en diversos países y autores.

Salvo las excepciones ya señaladas, hay una negación de la existencia del género y sus rasgos en la ficción en español, a pesar de que una revisión de fuentes apunta en la dirección opuesta. Esta característica resulta en que las pocas menciones al gótico parezcan una anomalía en la historia de la ficción en español, con el siguiente problema metodológico descrito por Aldana Reyes:

One of the main difficulties attending anyone aiming to explore the history of the Spanish Gothic is the country's reluctance to use this word to describe national outputs that would, if considered under the parameters by which the Gothic is measured in Anglophone countries, be found to be part of the canon in either content or intent (9).

Esto afecta tanto a literatura española como a la latinoamericana, si bien en el caso de la primera hay más información sobre el corpus, pues el término gótico ya es más empleado por la crítica; mientras que en el caso de la segunda se carece aún de una sistematización. De cualquier manera, el sesgo es sistemático, si bien los nuevos estudios hacen pensar en la posibilidad de su reconocimiento en las historias de la literatura.

La omisión del término "gótico", tanto como género como categoría descriptiva, es muy similar a lo que sucede con la fantasía y la ciencia ficción. Ambos géneros y términos se encuentran excluidos casi de manera total de la historia de la literatura. En el caso de la fantasía, existen textos diversos, algunos de autores canónicos, obras premiadas y éxitos de venta como La úlitima fada (1916) de Emilia 
Pardo Bazán; Merlín y Viviana (1930) de Benjamín Jarnés; Merlín y Familia (1955) de Álvaro Cunqueiro; Libro de caballerías (1957) de Joan Perucho; El unicornio (1965) de Manuel Mujica Láinez; La torre vigía (1971), Olvidado rey Gudú (1996) y Aranmanoth (2000) de Ana María Mature; Galaor (1972) de Hugo Hiriart; El rapto del Santo Grial (1993) de Paloma Díaz-Mas; La rosa de plata (1999) de Soledad Puertolas; buena parte de las obras de Laura Gallego escritas desde el año 2000, incluyendo los ciclos de las Crónicas de la torre y Memorias de Idhún; las obras de Rafáel Ábalos desde el 2000; Historia del rey transparente (2005) de Rosa Montero; la Saga de Tramórea (2005-2011) de Javier Negrete; jPor san Jorge! (2011) de Miguel Ángel Moleón Viana; Loba (2013) de Verónica Murguía; Los reinos de Grubmion (2015) de Soraya del Ángel Moreno, entre otros. Este recuento, aunque no es exhaustivo, basta para mostrar la gran presencia del género en español y representa la variedad y diversidad de la producción de fantasía en español.

De los listados en el párrafo anterior, el caso de Ana María Matute ilustra de manera paradigmática los problemas de las historias de la literatura con la omisión del término para designar el género. La autora es casi una figura omnipresente en los panoramas e historias de la literatura española del siglo XX. Además, en vida contó con múltiples reconocimientos importantes, por ejemplo, fue académica de número de la RAE y galardonada con el premio Cervantes en el año 2010. Con la excepción del estudio de Labanyi, su presencia es unánime en las secciones dedicadas a la novela de la posguerra española; sin embargo, no sucede lo mismo con sus obras de fantasía, donde destaca el caso de Olvidado rey Gudú. Esta novela fue la más exitosa de las obras de Matute, la más querida por la propia autora y la depositada en la Caja de las Letras del Instituto Cervantes, con motivo del premio homónimo (Gracia y Ródenas 675).

En general, la inclusión de la obra de Matute en las historias de la literatura se limita a las novelas de la posguerra, como en los trabajos José Carlos Mainer, o se centran en dicho período (Pérez; Molinaro), relegando las obras posteriores, las de fantasía, a simples menciones, que no siempre incluyen a Olvidado rey Gudú (Pérez 72). Otros estudios directamente minimizan las novelas de fantasía de la autora, sin aportar argumentos, más allá de valoraciones subjetivas, que podrían estar vinculadas al cambio de estética de sus novelas: "Ana María Matute’s (1925) early work was trully excellent .. . Her novels became over time more and more conventional, e.g. Olvidado rey Gudú” (Gullón 386). ${ }^{7}$ Las historias de la literatura que valora positivamente la novela tampoco identifican el género literario al que

7 Para una perspectiva detallada sobre el caso de Ana María Matute, véase (Gutiérrez Trápaga, “Olvidado rey Gudú y los olvidos metodológicos...”). 
pertenece de Gudú, como en el caso de Zahareas y Moix (1999 473). En una historia de 2011, se enuncia claramente la filiación de Gudú con la fantasía, pero con extraños matices:

Esta fantasía medievalizante tiene su origen un cuarto de siglo atrás, hacia 1970, cuando la escritora trasvasó sus temas de siempre a un entorno mítico medieval .. . No obedece, pues, en modo alguno, a la moda de ficción de espada y brujería auspiciada por la tardía difusión de El señor de los anillos de Tolkien o la vulgarización de la materia de Bretaña ofrecida por una película como Excalibur de John Boorman ... Estamos, en fin, en el terreno mixto del fairy tale y la ficción caballeresca y sentimental (Gracia y Ródenas 674-75).

Además de ser una de las pocas citas en una historia de la literatura que reconoce el género de la obra, llama también la atención que se niegue el vínculo literario de Gudú con Tolkien o con la ficción artúrica. Así, el caso de la novela más famosa y trascendente de Matute representa claramente la tendencia a ignorar y evitar el término fantasía, privilegiando otro tipo de obras, a pesar del éxito y la influencia en la narrativa en español. Por tanto, como sucede con el gótico, el no emplear la categoría de "fantasía" para describir una serie de obras dificultad su estudio individual, negando la existencia del género en español y la posibilidad de investigarlo y enmarcar sus obras de manera adecuada.

Si existen problemas para incluir o describir una de las novelas más exitosas de la fantasía en español como Gudú, no resulta extraño que el término "fantasía” esté casi ausente de las historias de la literatura. Cuando aparece, rara vez es para designar el género popular y por lo general se emplea como sinónimo de imaginación o como concepto psicológico y no literario. La Historia de Oviedo identifica a un grupo de autores como de fantasía: "Elizondo, Melo, Britto y otros" (Historia de la literatura hispanoamericana 4 418-420). En realidad, la definición seguida por Oviedo abarca tanto la fantasía como la literatura experimental:

Ciertos autores de nuestra época reaccionan ante ella concibiendo mundos del todo imaginarios, que les brindan la libertad estética para jugar con fantasmagorías e imágenes oníricas, explorar las puras posibilidades del lenguaje y producir obras que quieren ser, eminentemente, textos, invenciones verbales concebidas como alternativas al mundo real. El esfuerzo de algunos de estos autores supone un reflujo del espíritu vanguardista por su carácter experimental, en el que se entremezclan otras fórmulas (metaficción, postestructuralismo, etc.) más recientes (Historia de la literatura hispanoamericana 4 419). 
A pesar de este reconocimiento confuso, no se identifican más autores y obras pertenecientes a este género, como en el caso de Mujica Láinez, quien está incluido, pero no su novela El unicornio. La descripción de dicha obra apunta hacia la fantasía, pero no se enuncia explícitamente: "Mujica quiso ignorar por completo el prosaico presente y las formas literarias que reflejaban la realidad social . . . con una fuerte tendencia arcaizante: sus ideales estaban en la España medieval y aurisecular" (Historia de la literatura bispanoamericana 4 102). Así, aún en los poquísimos casos donde se llega a nombrar y reconocer obras del género, éstas aparecen como una minucia y mal identificadas, mostrando el sesgo crítico y metodológico. Luego, el primer paso para poder conocer el género, consistiría en nombrarlo, para poder identificar, clasificar y estudiar sus fuentes; así como tener una idea cuantitativa clara de su presencia real en la literatura en lengua española.

Nuevamente, sucede lo mismo con el caso de la ciencia ficción en español que con los otros dos géneros ya explorados: existen fuentes primarias y estudios especializados, pero las historias de la literatura no reconocen su existencia, ni emplean el término para describir obras individuales. En este caso, existen estudios panorámicos, números monográficos y diversas antologías que demuestran la existencia del género de manera abundante en español desde hace más de un siglo (Castro; Lockhart; Molina-Gavilán et al.; Haywood Ferreira; Kurlat Ares; Díez y Moreno). La lista de autores que han cultivado la ciencia ficción es enorme, por lo que únicamente enumero algunas de las plumas más famosas que han escrito páginas de ciencia ficción: Domingo Sarmiento, Rubén Darío, Miguel de Unamuno, Emilia Pardo Bazán, Azorín, Ramón Gómez dela Serna, Leopoldo Lugones, Amado Nervo, Julio Torri, Horacio Quiroga, Vicente Huidobro, Adolfo Bioy Casares, Jorge Luis Borges, Juan José Arreola, Hugo Correa, Carlos Fuentes, José Emilio Pacheco, Isabel Allende, Mario Levrero, Eduardo Mendoza, Elena Aldunate, Ana María Shua, Elia Barceló, César Aira, Carmen Boullosa, Rosa Montero, Alberto Chimal, Bernardo Fernández, entre otros. Entonces, es un género que se ha cultivado desde el siglo XIX y que se sigue desarrollando.

A pesar de la riqueza y vastedad del panorama de la ciencia ficción escrita en español, como se puede apreciar en la lista anterior e, inclusive, la existencia de numerosos estudios especializados, predomina la negación de la existencia o el desprecio del género en manuales e historia de la literatura:

In Latin America, perhaps more so than elsewhere, science fiction has long been considered to be a lesser form of literature. This, in spite of the fact that Latin American writers have long been practicing (since at least the eighteenth century) the genre as a means of cultural expression (Lockhart 8). 
Este comentario se puede hacer extensivo a la literatura española y, sin duda, representa en un alto porcentaje lo que sucede con la presentación del género en gran parte de las historias de la literatura. Tanto en el caso español como en latinoamericano, en buena parte de los trabajos revisados en esta investigación se repite la tendencia ya señalada para el gótico y la fantasía: no dedicar apartados a ese género y emplear parcamente la etiqueta genérica para describir obras individuales o rasgos autorales.

Varias historias simplemente no utilizan el término "ciencia ficción”, como si el género no existiera en español; así sucede en los casos de los trabajos de Gies (2004), Hart (2007), Labanyi (2010), Alonso (2010), Mainer (2010), González Echevarría (2012), ${ }^{8}$ por nombrar algunos casos. En otras instancias, el género sí que se reconoce para adjetivar obras o autores individuales. Por ejemplo, la Oxford History of the Spanish Novel reconoce la existencia de una obra, El Anacronópete (1887) de Enrique Gaspar y Rimbau (Álvarez-Castro 252). Por su parte, Espinasa en su trabajo sobre literatura mexicana reconoce a Rafael Bernal y a Hugo Hiriart como autores de este género (2015).

Dos obras, como ya se ha señalado, identifican claramente la existencia de géneros no realistas, incluyendo la ciencia ficción, las de Altisent y Oviedo. En el caso de la primera, la ciencia ficción no sólo es identificada por la editora del volumen, sino por varios de los autores de que participan en la publicación:

In response to the surge in market capitalism and consumer culture, and the rise in the disposable income of the average Spaniard, the 1980s saw a publishing boom, most notably in the so-called popular genres of science fiction, children's literature, detective fiction, and erotica (Molinaro 169).

De igual manera, en otros capítulos se identifican varias obras y autores, como la novela en catalán Mon mascle (1971) de Terenci Moix, Sin noticias de Gurb (1991) de Eduardo Mendoza o el género en gallego (Altisent, A Companion to the Twentieth-Century Spanish Novel 155, 180, 244). En el caso de Oviedo, además de señalar el vínculo de la ciencia ficción con lo fantástico, identifica obras y rasgos en tres autores

8 Esto coincide con lo expresado en la monumental y voluminosa The Cambridge History of Latin American Literature editada por el mismo autor y Enrique Pupo-Walker publicada en, fecha por la que no se incluyó esta historia en este trabajo. En los dos volúmenes dedicados a la literatura en español de esta obra apenas hay dos brevísimas menciones a la ciencia ficción: la primera para señalar la aparición de novelas del género a finales del XIX y la segunda para referirse a Las fuerzas extrañas de Leopoldo Lugones (Benítez-Rojo 468; González Echevarría y Pupo-Walker, The Cambridge History of Latin American Literature. 1. Discovery to Modernism 530). 
Adolfo Bioy Casares, Angélica Gorodischer y Manuel Puig (Historia de la literatura hispanoamericana 4 40, 269, 354). De cualquier forma, son pocas las menciones para una obra tan voluminosa y que reconoce la existencia del género desde el inicio.

El género de la ciencia ficción tiene un reconocimiento un poco mayor que el gótico o la fantasía, pero su presencia e identificación resulta aún muy deficiente. Como en los otros dos géneros aquí estudiados, la referencia a la ciencia ficción casi siempre se limita a breves descripciones en el plano individual, ya sea de algunos autores, ya sea de algunas obras. A diferencia de los que señalan los estudios especializados, la realidad es que en su mayoría la ciencia ficción no se considera como un género relevante por los manuales, historias y otros estudios de índole panorámico. Dos obras colectivas de reciente publicación sobre la historia del género en España y Latinoamérica (López-Pellisa; López-Pellisa y Kurlat Ares) demuestran la presencia y la abundancia del género, en espera de que los futuros estudios panorámica y de la historia de la literatura incluyan la ciencia ficción. Tan sólo estas dos obras, que no pretenden ser catálogos exhaustivos de fuentes, mencionan un corpus amplísimo de fuentes primarias que abarca la novela, el cuento, la poesía, el teatro, la narrativa gráfica, el cine y la televisión. Para Latinoamérica se cuenta por lo menos con 1250 fuentes (Molina-Gavilán et al.; López-Pellisa y Kurlat Ares).9 Para el caso español, si nos limitamos a la narrativa, como la forma dominante, el volumen editado por López-Pellisa y Kurlat Ares mencionan más de 150 obras, con varias acotaciones que sugieren que este número es tan sólo una fracción.10 Estos estudios enfatizan la importancia diacrónica del género durante más de dos siglo y zanjan cualquier posible duda sobre la existencia y la importancia de la ciencia ficción en español, sin bien aún falta por hacer un catálogo detallado de fuentes primarias para pensar en una aproximación profunda de distant reading sobre estas y no sobre su articulación en la historia de las literaturas hispánicas.

9 Considérese que el estudio de Molina Gavilán et al. no abarca los últimos quince años y que el de López-Pellisa y Kurlat Ares es el volumen uno, en el cual no se abarca el último medio siglo.

10 Por ejemplo: “. . . la revista más longeva de ciencia ficción aparecida en España, Nueva Dimensión, la cual se especializó en la difusión de relatos. Publicada entre 1968 y 1983, alcanzó un total de 148 números y 12 ejemplares extra . . ." y “. . . la obra cuentística de estos autores es enorme en su conjunto, dando lugar a una larga lista de escritores de calidad muy heterogénea y en general de reducida obra particular. Son demasiados los nombres a citar" (Peregrina Castaños 137 y 140). 


\section{Conclusiones}

Existe un enorme contraste entre la presentación de los tres géneros populares estudiados en las historias de la literatura y la información sobre el corpus que conforma estos géneros obtenida a partir de una revisión de las fuentes primarias. Al examinar múltiples historias de la literatura, el panorama legado sobre estos tres géneros en la literatura hispánica es que su existencia es nula o marginal, en el mejor de los casos. Así, de manera implícita, la tendencia en estos trabajos es negar la existencia de estos géneros y, en otros casos cuando se llega a reconocer la poética de estos géneros en algunos autores, se suele evitar el empleo de los términos "gótico", "fantasía" o "ciencia ficción", como en el caso de Quiroga con el gótico o Matute y la fantasía. Las pocas veces que sí se identifica y se reconoce a los tres géneros no suele haber una sección dedicada a éstos, ni un seguimiento detallado de las fuentes existentes. Así, en este trabajo se ha encontrado una muestra clara del proceso de génesis de las historias de la literatura, que pasa más por la intertextualidad al interior de propio género académico que por la revisión de fuentes del objeto de estudio:

... literary histories are made out of literary histories. Not only their classifications but also their plots are derived from previous histories of the same field .. . The authorities of a literary historian rests on other authorities, which are in fact, no more authoritative than the present one (Perkins 73).

Es decir, las historias de la literatura han repetido y heredado los sesgos de las historias previas más que partir de un trabajo de revisión e identificación de fuentes primarias, en lo que respecta a los tres géneros aquí estudiados.

Una aproximación a los mismos géneros en las historias de la literatura partiendo de una metodología de lectura distante o distant reading permite observar la omisión en dichos estudios y enfatiza la necesidad de volver a las fuentes y abarcar un corpus más amplio, empezando por reconocer la clasificación genérica de las obras, sin depender de los sesgos del relato prestablecido. En primer lugar, la revisión del corpus, aún sin ser exhaustiva, muestra que existe una enorme abundancia y variedad de obras, elaborada en distintas geografías y momentos, lo que prueba no sólo la existencia de dichos géneros, sino la presencia una tradición de más de dos siglos. Además, destaca que en la nómina de autores que han dedicado páginas a los tres géneros hay autores y obras famosas, canónicas y premiadas, por lo que dicha situación no se puede atribuir por completo a un problema de acceso o desconocimiento de fuentes. También existen importantes estudios especializados 
sobre estos textos; sin embargo, todo lo anterior no se refleja en las historias de la literatura y en la visión general que éstas dan sobre la literatura escrita en español.

A partir de este caso de estudio, se aprecia la necesidad de revisar la metodología de la historia de la literatura como manera de replantear la existencia, lugar y relevancia de las fuentes y los géneros literarios estudiados. La aproximación con distant reading permite detectar una serie de sesgos y distorsiones metodológicas de distinta índole, ya sean de metodología, de preferencia estética o ideológica, en los estudios literarios. En este caso, la distorsión no sólo se manifiesta en ignorar o excluir fuentes y géneros, sino en el nivel léxico, eliminando la posibilidad de la descripción e identificación más elemental. Con esto quedan borrados de manera sistemática los géneros referidos y también la posibilidad de estudiar muchas de sus obras individuales, al no poderlas definir como objeto de estudio válidos de la disciplina o presentarlas con una etiqueta ajena que distorsiona su filiación genérica. En ese sentido, examinar la información literaria a partir de una perspectiva amplia e incluyendo evidencia cuantitativa muestra una serie de factores, que pueden ser dominantes en un sistema literario, pero que han recibido poca atención crítica. De cualquier forma, para realmente poder realizar un estudio completo de lectura distante con las fuentes y no con la historia de la crítica es fundamental poder dar el primer paso: reconocer la existencia de dichas fuentes y su filiación genérica. Sólo entonces, será posible pensar en estudios más ambiciosos y precisos para considerar la presencia y la transformación de estos géneros en español. Dado el sinnúmero de obras que componen estos tres géneros, una aproximación por medio de distant reading sería necesaria para poder manejar dicho volumen textual.

Los tres géneros considerados comparten, además de su popularidad e influencia cultural, rasgos de poética que los acercan, como la presencia de elementos extraordinarios o sobrenaturales y una distancia importante con los postulados realistas o miméticos. Estos rasgos parecen centrales para ser la base del sesgo que explica, pero no justificar, su exclusión, pues, a partir del close reading, las historias de la literatura han dado preferencia casi absoluta a las obras de corte mimético o realista. El resto queda desechado o se muestra como una rareza o un asunto marginal, sin importar que la cantidad de fuentes apunte en dirección opuesta. En cambio, otras metodologías, como el distant reading, reforzarían la necesidad de incorporar y estudiar el gótico, la fantasía y la ciencia ficción a la historia de la literatura para dar cuenta de la existencia de otras tradiciones literarias más allá del realismo, lo verosímil o lo mimético. Luego, sería necesario revisar la presencia o ausencia otros géneros, más allá de los vistos en este artículo, así como repensar la metodología y el manejo de fuentes de las historias de la literatura. Con ello se 
plantea la posibilidad de trascender los sesgos de visiones limitadas a unas pocas obras y unos pocos autores, al incluir una perspectiva sin estos sesgos. Entonces, se podrá tener una representación más amplia y compleja de la diversidad literaria en español, más allá de las preferencias estéticas personales de los críticos, y completar o confrontar los resultados de otras metodologías.

Según lo señalado por Barthes al inicio de este trabajo, seguir la propuesta de Moretti implica modificar la metodología y los procesos de síntesis con los cuales se ha construido la historia de la literatura y el canon. Con esto se puede tener una visión más amplia del sistema y campo literario, dando cuenta también de las preferencias editoriales y lectoras y no sólo las autorales. Si bien Moretti señala que este cambio metodológico conlleva la pérdida del enfoque textual. En realidad, se puede plantear como una perspectiva que puede complementar o replantear toda la información y conocimiento derivado del enfoque textual ya existente. Es decir, la adopción del distant reading o de información cuantitativa no tiene implica el olvido forzoso del close reading. Por ejemplo, en el caso de los tres géneros revisados es claro el sesgo y la omisión de las historias de la literatura. Más allá de argumentar la necesidad de incorporar dichos géneros a la historia de la literatura, el distant reading permitiría ampliar su estudio, pero, dado que las fuentes de estos tres géneros han sido ignoradas o mal clasificadas, también es necesario construir los estudios faltantes por medio del close reading. Entonces, falta replantear la manera en que se elaborar las historias de la literatura y se descartan u omiten fuentes primarias y géneros completos, abundantes en obras y con siglos de existencia, que nos llevan a visiones muy parciales y sesgadas desde un inicio. 


\section{Obras citadas}

Aldana Reyes, Xavier. Spanish Gothic. National Identity, Collaboration and Cultural Adaptation. Londres: Palgrave Macmillan, 2017.

Alonso, Cecilio. Historia de la literatura española. 5. Hacia una literatura nacional 18001900. Editado por José Carlos Mainer, Barcelona: Crítica, 2010.

Altisent, Martha Eulalia, editor. A Companion to the Twentieth-Century Spanish Novel. Woodbridge: Tamesis, 2008.

. "Introduction". A Companion to the Twentieth-Century Spanish Novel. Woodbridge: Tamesis, 2008. 1-14.

Álvarez Barrientos, Joaquín. "Eighteenth-century prose writing". The Cambridge History of Spanish Literature, editado por David T. Gies. Cambridge: Cambridge University Press, 2004. 314-22.

. "The Spanish novel in the Eighteenth Century". A History of the Spanish Novel, editado por J. A. Garrido Ardila. Oxford University Press, 2015. 195-215.

Álvarez-Castro, Luis. "The Naturalist Novel in Spain. Nationalism Morality, and Aesthetics". A History of the Spanish Novel, editado por J. A. Garrido Ardila. Oxford: Oxford University Press, 2015. 234-254.

Barthes, Roland. "Histoire et littérature: à propos de Racine". Annales. Économies, Sociétés, Civilisations, vol. 15, núm. 3 (1960): 524-37.

Benitez-Rojo, Antonio. "The Nineteenth-Century Spanish American Novel”. The Cambridge History of Latin American Literature 1. Discovery to Modernism, editado por Roberto Gonzalez Echevarría y Enrique Pupo-Walker, vol. 1. Cambridge: Cambridge University Press, 1996. 401-89.

Bergero, Adriana J., y Elizabeth A. Marchant. "Feminist Insurrections: From Queiroz and Castellanos to Morejón, Poniatowska, Valenzuela, and Eltit". $A$ Companion to Latin American Literature and Culture, editado por Sara Castro-Klaren. Oxford y Malden, MA: Blackwell, 2008. 509-30.

Casanova-Vizcaíno, Sandra, e Inés Ordiz. Latin American Gothic in Literature and Culture. Nueva York: Routledge, 2018.

Castro-Klaren, Sara, editor. A Companion to Latin American Literature and Culture. Oxford y Malden, MA: Blackwell, 2008. 
Cortázar, Julio. "Notas sobre lo gótico en el Rio de la Plata". Caravelle. Cabiers du monde hispanique et luso-brésilien, vol. 25, núm. 1 (1975): 145-51.

Díez, Julián y Fernando Ángel Moreno. Historia y antología de la ciencia ficción española. Madrid: Cátedra, 2014.

Edwards, Justin D. y Sandra Guardini Vasconcelos. Tropical Gothic in Literature and Culture: The Americas. Nueva York: Routledge, 2016.

Espinasa, José María. Historia minima de la literatura mexicana en el siglo XX. México: El Colegio de Mexico, 2015.

Gies, David T. The Cambridge History of Spanish Literature. Cambridge: Cambridge University Press, 2004.

González Echevarría, Roberto. Modern Latin American Literature. A Very Short Introduction. Oxford: Oxford University Press, 2012.

González Echevarría, Roberto y Enrique Pupo-Walker, editores. The Cambridge History of Latin American Literature 1. Discovery to Modernism. Cambridge: Cambridge University Press, 1996. , editores. The Cambridge History of Latin American Literature 2: The Twentieth Century. Cambridge: Cambridge University Press, 1996.

Gracia, Jordi, y Domingo Ródenas. Historia de la literatura española 7. Derrota y restitución de la modernidad 1929-2010, editado por José Carlos Maine. Madrid: Crítica, 2011.

Gullón, Germán. "The Banquet Years of the Spanish Novel. By Invitation Only". A History of the Spanish Novel, editado por J. A. Garrido Ardila, Oxford: Oxford University Press, 2015. 376-90.

Gutiérrez Trápaga, Daniel. “La narrativa gótica en la historia de la literatura española: un problema de valoración de fuentes y metodología de la investigación". Bulletin of Spanish Studies: Hispanic Studies and Research on Spain, Portugal and Latin America, vol. 96, num. 9 (2019): 1413-1432.

“"Olvidado rey Gudú y los olvidos metodológicos de las fuentes de la novela de fantasía y la magia en la historia de la literatura española”, Storyca: Magia, brujería, Inquisición (dir. Antonio Huertas Morales), vol. 10 (2019): 33-46.

Hart, Stephen M. A Companion to Latin American Literature. Woodbribge: Tamesis, 2007. 
Haywood Ferreira, Rachel. The Emergence of Latin American Science Fiction. Middletown, Connecticut: Wesleyan University Press, 2011.

Hogle, Jerrold E. "Introduction: The Gothic in Western Culture". The Cambridge Companion to Gothic Fiction, editado por Jerrold E. Hogle. Cambridge: Cambridge University Press, 2002. 1-20.

Kurlat Ares, Silvia. "La ciencia ficción en América Latina: entre la mitología experimental y lo que vendrá”. Revista Iberoamericana (Pittsburgh). Special Issue, vol. LXXVIII, núm. 238-239, junio (2012): 15-22.

Labanyi, Jo. Spanish Literature: A Very Short Introduction. Oxford: Oxford University Press, 2010.

Lockhart, Darrell B., editor. Latin American Science Fiction Writers: An A-to-Z Guide. Westport: Greenwood Press, 2004.

López Castro, Ramón. Expedición a la ciencia ficción mexicana. México: Lectorum, 2001.

López-Pellisa, Teresa, editora. Historia de la ciencia ficción en la cultura española. Madrid y Frankfurt am Main: Vervuert-Iberoamericana, 2018.

López-Pellisa, Teresa y Silvia G. Kurlat Ares, editoras y directoras. Historia de la ciencia ficción latinoamericana I. Desde los origenes hasta la modernidad. Madrid y Frankfurt am Main: Vervuert-Iberoamericana, 2020.

López Santos, Miriam. La novela gótica en España (1788-1833). Vigo: Academia del Hispanismo, 2010.

Mainer, José Carlos. Historia de la literatura española. 6. Modernidad y nacionalismo: 1900 1939. Madrid: Crítica, 2010.

. Historia mínima de la literatura española. México: El Colegio de México y Turner, 2014.

Mendlesohn, Farah. Rhetorics of Fantasy. Middletown, Connecticut Wesleyan University Press, 2013.

Molina-Gavilán, Yolanda, et al. "Chronology of Latin American Science Fiction, 1775-2005”. Science Fiction Studies, vol. 34, núm. 3 (2007): 369-431.

Molinaro, Nina L., editor. "Narrating Women in the Post-War Spanish Novel". A Companion to the Twentieth-Century Spanish Novel, Woodbridge: Tamesis, 2008. 16173. 
Moreno, Fernando Ángel. Teoría de la literatura de ciencia ficción. Poética y retórica de lo prospectivo. Vigo: Portal, 2010.

Moretti, Franco. "Conjectures on World Literature". Distant Reading. Londres y Nueva York: Verso Books, 2013. 43-62.

- "The Slaughterhouse of Literature". Distant Reading. Londres y Nueva York: Verso Books, 2013. 63-89.

Oviedo, José Miguel. Historia de la literatura hispanoamericana 3. Posmodernismo, Vanguardia, Regionalismo. Madrid: Alianza Editorial, 2001.

- Historia de la literatura hispanoamericana 4. De Borges al presente. Madrid: Alianza Editorial, 2001.

Peregrina Castaños, Mikel. "Narrativa 1953-1980". Historia de la ciencia ficción en la cultura española, editado por Teresa López-Pellisa. Madrid y Frankfurt am Main: Vervuert-Iberoamericana, 2018. 123-150.

Pérez, Janet, editor. "The Social Realist Novel”. A Companion to the Twentieth-Century Spanish Novel. Woodbridge: Tamesis, 2008. 60-74.

Perkins, David. “Literary Classifications: How Have They Been Made?”. Is Literary History Possible? Baltimore y Londres: Johns Hopkins University Press, 1993. 61-84.

Pupo-Walker, Enrique. "The Brief Narrative in Spanish America: 1835-1915.” The Cambridge History of Latin American Literature 1. Discovery to Modernism, editado por Roberto Gonzalez Echevarría y Enrique Pupo-Walker, vol. 1. Cambridge: Cambridge University Press, 1996. 490-535.

Roberts, Adam. "Gothic and Horror Fiction". Cambridge Companion to Fantasy Literature, editado por Farah Mendlesohn y Edward James. Cambridge: Cambridge University Press, 2012. 21-35.

Seed, David. Science Fiction: A Very Short Introduction. Oxford: Oxford University Press, 2011.

Sumillera, Rocío G. "A vueltas con la periodización literaria”. Lingüistica y Literatura, vol. 74 (2018): 91-109.

Tolkien, J. R. R. On Fairy-Stories. Expanded Edition, with Commentary and Notes. Editado por Verlyn Flieger y Douglas A. Anderson, Londres: Harper Collins, 2014. 
Sesgos de las historias de la literatura: tres géneros narrativos populares...

Daniel Gutiérrez Trápaga

Turner, Harriet, y Adelaida López de Martínez, editores. The Cambridge Companion to the Spanish Novel: From 1600 to the Present. Cambridge: Cambridge University Press, 2003.

Zahareas, Anthony N., y Ana María Moix. "Ana María Matute". Historia y crítica de la literatura española. Época contemporánea 1939-1975. Primer suplemento, editado por Santos Sanz Villanueva, vol. 8/1. Barcelona: Crítica, 1999. 469-74. 